\title{
ON RANDOM ITERATED FUNCTION SYSTEMS WITH GREYSCALE MAPS
}

\author{
Matthew Demers ${ }^{1}$, Herb Kunze ${ }^{\bowtie}, 2$ And Davide LA TORRE ${ }^{3}$ \\ ${ }^{1}$ University of Guelph, Department of Mathematics \& Statistics, Guelph, Ontario, Canada; ${ }^{2}$ University of \\ Guelph, Department of Mathematics \& Statistics, Guelph, Ontario, Canada; ${ }^{3}$ University of Milan, Department \\ of Economics, Business and Statistics, Milan, Italy \\ e-mail: mdemers@uoguelph.ca; hkunze@uoguelph.ca; davide.latorre@unimi.it \\ (Received January 18, 2012; revised April 27, 2012; accepted May 8, 2012)
}

\begin{abstract}
In the theory of Iterated Function Systems (IFSs) it is known that one can find an IFS with greyscale maps (IFSM) to approximate any target signal or image with arbitrary precision, and a systematic approach for doing so was described. In this paper, we extend these ideas to the framework of random IFSM operators. We consider the situation where one has many noisy observations of a particular target signal and show that the greyscale map parameters for each individual observation inherit the noise distribution of the observation. We provide illustrative examples.
\end{abstract}

Keywords: random fixed point equations, random iterated function systems, collage theorem.

\section{INTRODUCTION}

In fractal imaging compression and coding based on Generalized Fractal Transforms (GFT), one seeks to approximate a target image or signal by the fixed points of a contractive fractal transform operator. The usual formulation involves a fixed set of geometric contraction maps along with a corresponding set of greyscale adjustment maps. The inverse problem requires one to find the best greyscale map parameters for a given target image. The solution process is referred to as "collage coding" because of the visual effect of the contraction maps; it applies the collage theorem, a simple consequence of Banach's fixed point theorem. The approximation of the target image or signal can be generated through iteration of the fractal transform (see Hutchinson, 1981; Barnsley et al., 1985; Barnsley and Demko, 1985; Barnsley, 1989; Barnsley and Hurd, 1993; Forte and Vrscay, 1995; 1999; Iacus and La Torre, 2005b;a; Kunze et al., 2008; La Torre et al., 2009; La Torre and Vrscay, 2011 for more details and applications).

In Forte and Vrscay (1995), the authors showed that one can find an iterated function system with greyscale maps (IFSM) to approximate any target signal or image with arbitrary precision, and they provided a suboptimal but systematic approach for doing so.

The goal of this paper is to extend the work in Forte and Vrscay (1995) to the framework of random IFSM operators. We consider the situation where one has many noisy observations of a particular target signal and show that the greyscale map parameters for each individual observation inherit the noise distribution of the observation.

Prior to these results, we present several short background sections. First, we discuss the deterministic collage coding framework and quickly summarize the related IFSM setting. Then we present the random collage coding framework. Finally, we formulate the random IFSM operator framework and present some illustrative numerical examples.

\section{FIXED POINT EQUATIONS AND COLLAGE THEOREM}

In this section, we present some basic contraction map results that will be used in later sections. Let $\left(X, d_{X}\right)$ denote a complete metric space. Then $T: X \rightarrow$ $X$ is contractive if there exists a $c \in[0,1)$ such that

$$
d_{X}(T x, T y) \leq c d_{X}(x, y) \text { for all } x, y \in X .
$$

We normally refer to the infimum of all $c$ values satisfying Eq. 1 as the contraction factor of $T$.

Theorem 1 (Banach): Let $\left(X, d_{X}\right)$ be a complete metric space. Also let $T: X \rightarrow X$ be a contraction mapping with contraction factor $c \in[0,1)$. Then there exists a unique $\bar{x} \in X$ such that $\bar{x}=T \bar{x}$. Moreover, for any $x \in X, d_{X}\left(T^{n} x, \bar{x}\right) \rightarrow 0$ as $n \rightarrow \infty$.

Theorem 2 (Continuity theorem for fixed points, Centore and Vrscay, 1994): Let $\left(X, d_{X}\right)$ be a complete metric space and $T_{1}, T_{2}$ be two contractive mappings 
with contraction factors $c_{1}$ and $c_{2}$ and fixed points $\bar{y}_{1}$ and $\bar{y}_{2}$, respectively. Then

$$
d_{X}\left(\bar{y}_{1}, \bar{y}_{2}\right) \leq \frac{1}{1-\min \left\{c_{1}, c_{2}\right\}} d_{X, \sup }\left(T_{1}, T_{2}\right)
$$

where

$$
d_{X, \sup }\left(T_{1}, T_{2}\right)=\sup _{x \in X} d_{X}\left(T_{1}(x), T_{2}(x)\right)
$$

Banach's fixed point theorem provides a mechanism to solve the forward problem of the fixed point equation $x=T x$ : Given a contraction $T$, construct, or at least approximate, its fixed point $\bar{x}$. And the continuity theorem establishes that small changes in a contraction mapping produce small changes in the associated fixed points.

A formal mathematical inverse problem associated with the fixed point equation $x=T x$ is: Given a target element $x$ and an $\varepsilon>0$, find a contraction map $T(\varepsilon)$ (perhaps from a suitable family of operators) with fixed point $\bar{x}(\varepsilon)$ such that $d(x, \bar{x}(\varepsilon))<\varepsilon$. In the case that one is able to solve such an inverse problem to arbitrary precision, i.e., $\varepsilon \rightarrow 0$, then one may identify the target $x$ as the fixed point of a contractive operator $T$ on $X$.

In practical applications, however, it is not generally possible to find such solutions to arbitrary accuracy nor is it even feasible to search for such contraction maps. Instead, one makes use of the following result, which is a simple consequence of Banach's fixed point theorem.

Theorem 3 ("Collage theorem", Barnsley et al., 1985): Let $(X, d)$ be a complete metric space and $T: X \rightarrow X$ a contraction mapping with contraction factor $c \in[0,1)$. Then for any $x \in X$,

$$
d(x, \bar{x}) \leq \frac{1}{1-c} d(x, T x),
$$

where $\bar{x}$ is the fixed point of $T$.

The approximation error $d(x, \bar{x})$ is bounded above by the so-called collage distance $d(x, T x)$. Most practical methods of solving such inverse problems, for example, fractal image coding (Fisher, 1995; Lu, 2003), search for an operator $T$ for which the collage distance is as small as possible, while ensuring that $c$ is sufficiently far away from one. In other words, these methods seek an operator $T$ that maps the target $x$ as close as possible to itself. This inverse problem solution procedure, often referred to as collage coding, is most often performed by considering a parametrized family of contraction maps $T_{\lambda}, \lambda \in \Lambda \subset \mathbb{R}^{n}$, and then minimizing the collage distance $d\left(x, T_{\lambda} x\right)$.

Finally, we mention another interesting result which is a simple consequence of Banach's fixed point theorem.

Theorem 4 ("Anti-collage theorem", Vrscay and Saupe, 1999): Let $(X, d)$ be a complete metric space and $T: X \rightarrow X$ a contraction mapping with contraction factor $c \in[0,1)$. Then for any $x \in X$,

$$
d(x, \bar{x}) \geq \frac{1}{1+c} d(x, T x),
$$

where $\bar{x}$ is the fixed point of $T$.

\section{FORMAL SOLUTION TO THE INVERSE PROBLEM FOR ITERATED FUNCTION SYSTEMS WITH GREYSCALE MAPS}

The method of iterated function systems with greyscale maps (IFSM), as formulated by Forte and Vrscay (1995), can be used to approximate a given element $u$ of $L^{2}([0,1])$. We consider the case in which $u:[0,1] \rightarrow[0,1]$ and the space

$$
X=\left\{u:[0,1] \rightarrow[0,1], u \in L^{2}[0,1]\right\} .
$$

The ingredients of an $N$-map IFSM on $X$ are (Forte and Vrscay, 1995; 1999)

1. a set of $N$ contractive mappings $w=$ $\left\{w_{1}, w_{2}, \ldots, w_{N}\right\}, w_{i}:[0,1] \rightarrow[0,1]$, satisfying the covering condition $\bigcup_{i=1}^{N} w_{i}([0,1])=[0,1]$, and most often affine in form:

$$
w_{i}(x)=s_{i} x+a_{i}, \quad 0 \leq s_{i}<1, \quad i=1,2, \ldots, N
$$

2. a set of associated functions - the greyscale maps $\phi=\left\{\phi_{1}, \phi_{2}, \ldots, \phi_{N}\right\}, \phi_{i}: \mathbb{R} \rightarrow \mathbb{R}$. Affine maps are usually employed:

$$
\phi_{i}(t)=\alpha_{i} t+\beta_{i},
$$

with the conditions

$$
\alpha_{i}, \beta_{i} \in[0,1]
$$

and

$$
0 \leq \sum_{i=1}^{N} \alpha_{i}+\beta_{i}<1
$$


Associated with the $N$-map IFSM $(w, \phi)$ is the fractal transform operator $T$, the action of which on a function $u \in X$ is given by

$$
(T u)(x)=\sum_{i=1}^{N} \phi_{i}\left(u\left(w_{i}^{-1}(x)\right)\right),
$$

where the prime means that the sum operates on all those terms for which $w_{i}^{-1}$ is defined.

Theorem 5 (Forte and Vrscay, 1995): $T: X \rightarrow X$ and for any $u, v \in X$ we have

$$
d_{2}(T u, T v) \leq C d_{2}(u, v)
$$

where

$$
C=\sum_{i=1}^{N} s_{i}^{\frac{1}{2}} \alpha_{i}
$$

When $C<1$, then $T$ is contractive on $X$, implying the existence of a unique fixed point $\bar{u} \in X$ such that $\bar{u}=T \bar{u}$.

The squared collage distance function associated with an $N$-map IFSM may be written as a quadratic form,

$$
\Delta^{2}=z^{T} A z+b^{T} z+c,
$$

where $z=\left(\alpha_{1}, \ldots \alpha_{k}, \beta_{1}, \ldots, \beta_{k}\right)$. We observe that in the special that the sets $w_{i}([0,1]), i=1, \ldots, N$, are nonoverlapping, for each $i$ the values of $\alpha_{i}$ and $\beta_{i}$ that minimize $\Delta^{2}$ can be determined by solving a separate minimization problem using least squares.

The inverse problem associated with IFSM can, in principle, be solved to arbitrary accuracy, using a procedure defined in Forte and Vrscay (1995). The maps $w_{k}$ are chosen from an infinite set $W$ of fixed affine contraction maps on $[0,1]$ which satisfy the following properties.

Definition 1 We say that $W$ generates an $m$-dense and nonoverlapping family $A$ of subsets of I if for every $\varepsilon>0$ and every $B \subset I$ there exists a finite set of integers $i_{k}, i_{k} \geq 1,1 \leq k \leq N$, such that

$$
\begin{aligned}
& \text { (i) } A=\cup_{k=1}^{N} w_{i_{k}}(I) \subset B, \\
& \text { (ii) } m(B \backslash A)<\varepsilon \text {, and } \\
& \text { (iii) } m\left(w_{i_{k}}(I) \cap w_{i_{l}}(I)\right)=0 \text { if } k \neq l \text {, }
\end{aligned}
$$

where $m$ denotes Lebesgue measure.

Let

$$
W^{N}=\left\{w_{1}, \ldots w_{N}\right\}
$$

be the $N$ truncations of $w$. Let $\Phi^{N}=\left\{\phi_{1}, \ldots, \phi_{N}\right\}$ be the $N$-vector of affine grey level maps. Let $z_{N}$ be the solution of the previous quadratic optimization problem and $\Delta_{N, \text { min }}^{2}=\Delta_{N}^{2}\left(z_{N}\right)$. In Forte and Vrscay (1995), the following result was proved.

\section{Theorem 6}

$$
\Delta_{N, \min }^{2} \rightarrow 0 \text { as } N \rightarrow \infty .
$$

Using the Collage Theorem, the inverse problem may be solved to arbitrary accuracy. A practical choice for the contraction maps $w$ on $X=[0,1]$ is

$$
w_{i j}(x)=2^{-i}(x+j-1), i=1,2, \ldots, j=1,2, \ldots, 2^{i} .
$$

\section{RANDOM FIXED POINT EQUATIONS}

Let $(\Omega, \mathscr{F}, \mathbb{P})$ denote a probability space and $(X, d)$ a metric space. A mapping $T: \Omega \times X \rightarrow X$ is called a random operator if for any $x \in X$ the function $T(., x)$ is measurable. A measurable mapping $x: \Omega \rightarrow$ $X$ is called a random fixed point of a random operator $T$ if $x$ is a solution of the equation

$$
T(w, x(w))=x(w) .
$$

for a.e. $\omega \in \Omega$. A random operator $T$ is called continuous (Lipschitz, contraction) if for a.e. $w \in$ $\Omega$ we have that $T(w,$.$) is continuous (Lipschitz,$ contraction). There are many papers in the literature that deal with such equations for single-valued and setvalued random operators (see Bharucha-Reid, 1972; Itoh, 1979; Papageorgiou, 1988; Lin, 1995; Liu, 1997; Shahzad, 2001; 2004a;b). Solutions of random fixed point equations and inclusions are usually produced by means of stochastic generalizations of classical fixed point theory.

Let $(\Omega, \mathscr{F}, \mathbb{P})$ be a probability space and let $\left(X, d_{X}\right)$ be a complete metric space. Let $T: \Omega \times X \rightarrow X$ be a given operator. We look for the solution of the equation

$$
T(\omega, x(\omega))=x(\omega)
$$

for a.e. $\omega \in \Omega \backslash A$ and $\mathbb{P}(A)=0$. Suppose that the operator $T$ satisfies the inequality

$$
d(T(\omega, x), T(\omega, y)) \leq c(\omega) d(x, y),
$$

where $c(\omega): \Omega \rightarrow X$. When $T$ satisfies this property, we say that $T$ is a $c(\omega)$-Lipschitz operator. If $c(\omega) \leq$ $c<1$ a.e., with $\omega \in \Omega \backslash A$ and $\mathbb{P}(A)=0$ then we say that $T$ is a $c(\omega)$-contraction. In this case for $\omega \in$ $\Omega \backslash A$ there exists a unique point $x(\omega) \in X$. It is clear that the uniqueness makes sense except for sets of measure zero. Once again, the inverse problem can be formulated as: Given a function $x: \Omega \rightarrow X$ and a family of operators $T_{\lambda}: \Omega \times X \rightarrow X$, find $\lambda$ such that $x$ is the solution of random fixed point equation

$$
T_{\lambda}(\omega, x(\omega))=x(\omega) .
$$

We may now state the following random analogs to Theorems 1-4. 
Corollary 1 ("Regularity conditions"): Let $(\Omega, \mathscr{F}, \mathbb{P})$ be a probability space and $\left(X, d_{X}\right)$ be a complete metric space. Let $T: \Omega \times X \rightarrow X$ be a given $c(\omega)$-contraction. Then $d_{X}\left(x\left(\omega_{1}\right), x\left(\omega_{2}\right)\right) \leq$ $\frac{1}{1-c} \sup _{x \in X} d\left(T\left(\omega_{1}, x\right), T\left(\omega_{2}, x\right)\right)$

Corollary 2 ("Continuity Theorem"): Let $(\Omega, \mathscr{F}, \mathbb{P})$ be a probability space and let $\left(X, d_{X}\right)$ be a complete metric space. Let $T_{1}: \Omega \times X \rightarrow X$ and $T_{2}: \Omega \times X \rightarrow X$ be $c_{1}(\omega)$ - and $c_{2}(\omega)$-contractions respectively. Then

$$
\begin{aligned}
& d_{X}\left(x_{1}(\omega), x_{2}(\omega)\right) \leq \\
& \quad \frac{1}{1-\min \left\{c_{1}, c_{2}\right\}} \sup _{x \in X} d_{X}\left(T_{1}(\omega, x), T_{2}(\omega, x)\right) .
\end{aligned}
$$

Theorem 7 ("Collage Theorem"): Let $(\Omega, \mathscr{F}, \mathbb{P})$ be a probability space and let $\left(X, d_{X}\right)$ be a complete metric space. Let $T: \Omega \times X \rightarrow X$ be a given $c(\omega)$-contraction. Then

$$
\begin{array}{r}
\frac{1}{1+c} d_{X}(x(\omega), T(\omega, x(\omega))) \leq d_{X}(x(\omega), \bar{x}(\omega)) \leq \\
\frac{1}{1-c} d_{X}(x(\omega), T(\omega, x(\omega))),
\end{array}
$$

a.e. $\omega \in \Omega$, where $\bar{x}(\omega)$ is the solution of $T(\omega, \bar{x}(\omega))=$ $\bar{x}(\omega)$.

The above described approach to deal with random fixed point equations does not guarantee, in general, the measurability of the function $x: \Omega \rightarrow X$ with respect to the sigma algebra $\mathscr{F}$. In order to overcome this difficulty, we provide an abstract formulation of the same problem which guarantees the measurability of $x$.

Recall that $(X, d)$ is said to be a Polish space if it is a separable complete metric space. In random fixed point theory, separability plays an important role. Two important examples of Polish metric spaces which will be useful in the following are $C([a, b])$ and $L^{2}([a, b])$. Consider now the space $Y$ of all measurable functions $x: \Omega \rightarrow X$. If we define the operator $\tilde{T}: Y \rightarrow Y$ as $(\tilde{T} y)(\omega)=T(\omega, x(\omega))$ the solutions of this fixed point equation on $Y$ are the solutions of the random fixed point equation $T(\omega, x(\omega))=x(\omega)$. Suppose that the metric $d_{X}$ is bounded, that is $d_{X}\left(x_{1}, x_{2}\right) \leq K$ for all $x_{1}, x_{2} \in X$. So the function $\psi(\omega)=d_{X}\left(x_{1}(\omega), x_{2}(\omega)\right)$ : $\Omega \rightarrow \mathbb{R}$ is an element of $L^{1}(\Omega)$ for all $x_{1}, x_{2} \in \Omega$. We can then define on the space $Y$ the following function

$$
d_{Y}\left(x_{1}, x_{2}\right)=\int_{\Omega} d_{X}\left(x_{1}(\omega), x_{2}(\omega)\right) \mathrm{d} \omega .
$$

It can be shown that the space $\left(Y, d_{Y}\right)$ is a complete metric space. The following result holds.
Theorem 8 (Itoh, 1977): Let X be a Polish space, and $T: \Omega \times X \rightarrow X$ be a mapping such that for each $\omega \in \Omega$ the function $T(\omega,$.$) is c(\omega)$-Lipschitz and for each $x \in$ $X$ the function $T(., x)$ is measurable. Let $x: \Omega \rightarrow X$ be a measurable mapping; then the mapping $\xi: \Omega \rightarrow X$ defined by $\xi(\omega)=T(\omega, x(\omega))$ is measurable.

The previous theorem (Eq. 8) holds if $T(\omega, \cdot)$ is only continuous (see, for instance, Himmelberg, 1975).

Corollary 3 Let $X$ be a Polish space and $T: \Omega \times X \rightarrow$ $X$ be a mapping such that for each $\omega \in \Omega$ the function $T(\omega, \cdot)$ is a $c(\omega)$-contraction. Suppose that for each $x \in X$ the function $T(\cdot, x)$ is measurable. Then there exists a unique solution of the equation $\tilde{T} \bar{x}=\bar{x}$ that is $T(\omega, \bar{x}(\omega))=\bar{x}(\omega)$ for a.e. $\omega \in \Omega$.

In these results separability plays a crucial role. In fact using this hypothesis and Theorem 8 one can prove that the function $\xi(\omega)=T(\omega, x(\omega))$ is measurable, that is $\tilde{T}: Y \rightarrow Y$. Other random fixed point theorems for contraction mappings in Polish spaces can be found in Spaček (1955); Hanš (1957); Bharucha-Reid (1972). The inverse problem can be formulated as: Given a function $\bar{x}: \Omega \rightarrow X$ and a family of operators $\tilde{T}_{\lambda}: Y \rightarrow$ $Y$ find $\lambda$ such that $\bar{x}$ is the solution of random fixed point equation

$$
\tilde{T}_{\lambda} \bar{x}=\bar{x},
$$

that is,

$$
T_{\lambda}(\omega, \bar{x}(\omega))=\bar{x}(\omega)
$$

Now, with the collage and continuity theorems, we may state the following.

Corollary 4 Let $X$ be a Polish space and $T: \Omega \times X \rightarrow$ $X$ be a mapping such that for each $\omega \in \Omega$ the function $T(\omega, \cdot)$ is a $c(\omega)$-contraction. Suppose that for each $x \in X$ the function $T(\cdot, x)$ is measurable. Then for any $x \in Y$,

$$
\frac{1}{1+c} d_{Y}(x, \tilde{T} x) \leq d_{Y}(x, \bar{x}) \leq \frac{1}{1-c} d_{Y}(x, \tilde{T} x),
$$

where $\bar{x}$ is the fixed point of $\tilde{T}$, that is, $\bar{x}(\omega):=$ $T(\omega, \bar{x}(\omega))$. 


\section{FORMAL SOLUTION TO THE INVERSE PROBLEM FOR RANDOM ITERATED FUNCTION SYSTEMS WITH GREYSCALE MAPS}

We now formulate a Random IFSM fractal transform that is analogous to the deterministic fractal transform in Eq. 11.

Let $(\Omega, \mathscr{F}, \mathbb{P})$ be a probability space and consider now the following operator $T: X \times \Omega \rightarrow X$ defined by

$$
\begin{aligned}
T(\omega, u) & =(T u)(x) \\
& =\sum_{i=1}^{N} \alpha_{i}\left(u\left(w_{i}^{-1}(x)\right)\right)+\beta_{i}(\omega) \\
& =\left(T^{*} u\right)+\beta(\omega),
\end{aligned}
$$

where $\beta(\omega)=\sum_{i=1}^{N} \beta_{i}(\omega)$. That is, we suppose that the greyscale maps take the form

$$
\phi_{i}(\omega, t)=\alpha_{i} t+\beta_{i}(\omega),
$$

where $\beta_{i}$ are random variables with mean $\mu_{i}$ and $\left|\beta_{i}(\omega)\right|<\delta$ a.e. $\omega \in \Omega$, and that

$$
\alpha_{i}, \delta \in[0,1]
$$

with

$$
0 \leq \sum_{i=1}^{N}\left(\alpha_{i}+\delta\right)<1
$$

We randomize only the $\beta_{i}$ parameters since the randomness in the other term of $T(\omega, u)$ is transmitted by the function $u$. Let

$$
Y=\{u: \Omega \rightarrow X, u \text { is measurable }\}
$$

and consider the function

$$
\psi(\omega):=d_{X}\left(u_{1}(\omega), u_{2}(\omega)\right)=\left\|u_{1}(\omega)-u_{2}(\omega)\right\|_{2} .
$$

From the hypotheses it is clear that $\psi(\omega) \in L^{1}(\Omega)$. We know that $\left(Y, d_{Y}\right)$ is a complete metric space where

$$
d_{Y}\left(u_{1}, u_{2}\right)=\int_{\Omega}\left\|u_{1}(\omega)-u_{2}(\omega)\right\|_{2} d \omega .
$$

Obviously, the function $\xi(\omega):=\left(T^{*} u\right)(\omega)+\beta(\omega)$ belongs to $Y$ because $T^{*}$ is Lipschitz on $X$ and the $\beta$ is measurable. If we define $\tilde{T}: Y \rightarrow Y$ where $\tilde{T} u=\xi$ we have

$$
\begin{aligned}
& d_{Y}\left(\tilde{T} u_{1}, \tilde{T} u_{2}\right)= \\
& \int_{\Omega}\left\|\left(T^{*} u_{1}\right)(\omega)+\beta(\omega)-\left(T^{*} u_{2}\right)(\omega)-\beta(\omega)\right\|_{2} \mathrm{~d} \omega= \\
& \int_{\Omega}\left\|u_{1}(\omega)-u_{2}(\omega)\right\|_{2} \mathrm{~d} \omega=C d_{Y}\left(u_{1}, u_{2}\right) .
\end{aligned}
$$

So there exists $\bar{u} \in Y$ such that $\tilde{T} \bar{u}=\bar{u}$, that is, $\bar{u}(\omega)=$ $T(\omega, \bar{u}(\omega))$ for a.e. $\omega \in \Omega$. That is,

$$
\begin{aligned}
\bar{u}(\omega, x) & =\left(T^{*} \bar{u}(\omega)\right)(x)+\beta(\omega) \\
& =\sum_{i=1}^{N} \alpha_{i}\left(\bar{u}\left(\omega, w_{i}^{-1}(x)\right)\right)+\beta_{i}(\omega)
\end{aligned}
$$

for a.e. $x \in[0,1]$. The inverse problem of finding the random variables $\beta_{i}(\omega)$, perhaps to arbitrary accuracy, is note possible given the random nature of the problem. Instead, we suppose that the function $\bar{u}(\omega, x): \Omega \times[0,1] \rightarrow \mathbb{R}$ is integrable and let

$$
\bar{u}(x)=\int_{\Omega}[\bar{u}(\omega)](x) \mathrm{d} \omega=\int_{\Omega} \bar{u}(\omega, x) \mathrm{d} \omega .
$$

Thus Eq. 29 becomes

$$
\bar{u}(x)=\sum_{i=1}^{N} \alpha_{i}\left(\bar{u}\left(w_{i}^{-1}(x)\right)\right)+\mu_{i} .
$$

That is, the expectation value of $\bar{u}(\omega, x)$ is the solution of a deterministic $N$-map IFSM on $X$ (cf. Eq. 11). We observe in Eq. 31 another motivation for not randomizing the parameters $\alpha_{i}$ : the expectation of the product $\alpha_{i} u(\cdot)$ becomes complicated.

Suppose that we have $N$ observations, $u\left(\omega_{1}\right)$, $u\left(\omega_{2}\right), \ldots, u\left(\omega_{n}\right)$, of the variable $u(\omega)$. Choose a set of greyscale maps, as in Eq. 15. We collage code each observation, $u\left(\omega_{k}\right), k=1, \ldots, n$, to find the set of minimal collage parameters $\alpha_{i k}$ and $\beta_{i k}, k=1, \ldots, n$, $i=1, \ldots, N$. As a result of our earlier discussion, if the observations differ only by a uniform additive noise factor, we expect that the distribution of the $\beta_{i k}$ parameters, $k=1 \ldots, n$ will inherit that distribution, with mean $\mu_{i}$.

\section{NUMERICAL EXAMPLES}

This section provides several numerical examples which illustrate the above formulation and "mean property."

Example: We define the IFS maps

$w_{i j}(x)=2^{-i}(x+j-1), \quad i=1, \ldots, 4, j=1,2, \ldots, 2^{i}$, with associated greyscale maps $\phi_{i j}(t)=\alpha_{i j} t+\beta_{i j}$. In this example, we consider the target function

$$
u(x)=0.8 x^{2}+0.1 .
$$

We construct $N$ observations of the function by adding constant noise to it:

$$
u_{k}(x)=u(x)+v_{k}, \quad k=1, \ldots, N,
$$

where $\left|v_{k}\right|<0.1$ with mean zero. The picture in Fig. 1 presents ten realizations with constant and placedependent noise. 
Table 1. Greyscale map mean parameters $\alpha_{i, j}$ in the case of constant additive noise.

\begin{tabular}{cccc}
\hline coefficient & 50 & 100 & 200 \\
\hline$\alpha_{1,1}$ & 0.25000 & 0.25000 & 0.25000 \\
$\alpha_{1,2}$ & 0.25732 & 0.25730 & 0.25730 \\
$\alpha_{2,1}$ & 0.06250 & 0.06250 & 0.06250 \\
$\alpha_{2,2}$ & 0.06983 & 0.06982 & 0.06982 \\
$\alpha_{2,3}$ & 0.07715 & 0.07715 & 0.07715 \\
$\alpha_{2,4}$ & 0.08447 & 0.08447 & 0.08447 \\
$\alpha_{3,1}$ & 0.01563 & 0.01563 & 0.01563 \\
$\alpha_{3,2}$ & 0.02295 & 0.02295 & 0.02295 \\
$\alpha_{3,3}$ & 0.03028 & 0.03028 & 0.03028 \\
$\alpha_{3,4}$ & 0.03760 & 0.03760 & 0.03760 \\
$\alpha_{3,5}$ & 0.04493 & 0.04493 & 0.04493 \\
$\alpha_{3,6}$ & 0.05226 & 0.05226 & 0.05226 \\
$\alpha_{3,7}$ & 0.05958 & 0.05958 & 0.05958 \\
$\alpha_{3,8}$ & 0.06691 & 0.06691 & 0.06691 \\
$\alpha_{4,1}$ & 0.00391 & 0.00391 & 0.00391 \\
$\alpha_{4,2}$ & 0.01124 & 0.01124 & 0.01124 \\
$\alpha_{4,3}$ & 0.01857 & 0.01857 & 0.01857 \\
$\alpha_{4,4}$ & 0.02590 & 0.02590 & 0.02590 \\
$\alpha_{4,5}$ & 0.03323 & 0.03323 & 0.03323 \\
$\alpha_{4,6}$ & 0.04056 & 0.04056 & 0.04056 \\
$\alpha_{4,7}$ & 0.04790 & 0.04789 & 0.04789 \\
$\alpha_{4,8}$ & 0.05523 & 0.05523 & 0.05523 \\
$\alpha_{4,9}$ & 0.06256 & 0.06256 & 0.06256 \\
$\alpha_{4,10}$ & 0.06989 & 0.06989 & 0.06989 \\
$\alpha_{4,11}$ & 0.07722 & 0.07722 & 0.07722 \\
$\alpha_{4,12}$ & 0.08455 & 0.08455 & 0.08455 \\
$\alpha_{4,13}$ & 0.09188 & 0.09188 & 0.09188 \\
$\alpha_{4,14}$ & 0.09921 & 0.09921 & 0.09921 \\
$\alpha_{4,15}$ & 0.10655 & 0.10650 & 0.10650 \\
$\alpha_{4,16}$ & 0.11388 & 0.11390 & 0.11390 \\
\hline & & &
\end{tabular}
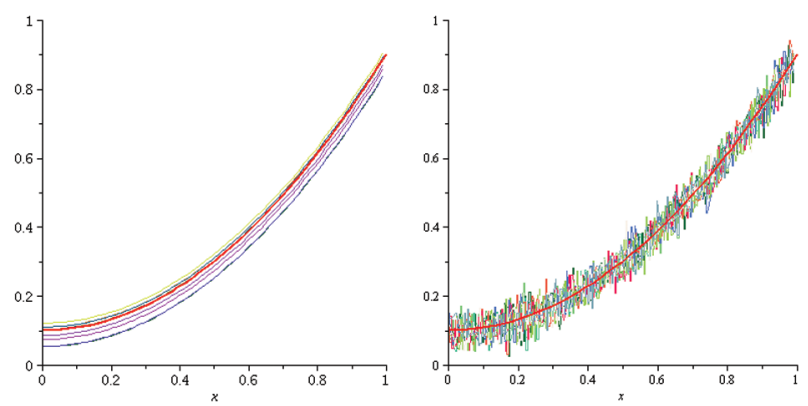

Fig. 1. Ten realizations for constant additive noise and place-dependent additive noise.

Tables 1 and 2 presents the mean value of each greyscale map parameter obtained by collage coding the separate realizations. The calculations were performed for 50, 100, and 200 realizations, using Maple.
Table 2. Greyscale map mean parameters $\beta_{i, j}$ in the case of constant additive noise.

\begin{tabular}{cccc}
\hline coefficient & 50 & 100 & 200 \\
\hline$\beta_{1,1}$ & 0.07105 & 0.07261 & 0.07199 \\
$\beta_{1,2}$ & 0.07157 & 0.07312 & 0.07250 \\
$\beta_{2,1}$ & 0.08881 & 0.09077 & 0.08998 \\
$\beta_{2,2}$ & 0.08948 & 0.09142 & 0.09065 \\
$\beta_{2,3}$ & 0.09054 & 0.09247 & 0.09170 \\
$\beta_{2,4}$ & 0.09200 & 0.09391 & 0.09315 \\
$\beta_{3,1}$ & 0.09325 & 0.09530 & 0.09448 \\
$\beta_{3,2}$ & 0.09451 & 0.09655 & 0.09573 \\
$\beta_{3,3}$ & 0.09733 & 0.09935 & 0.09854 \\
$\beta_{3,4}$ & 0.10171 & 0.10372 & 0.10292 \\
$\beta_{3,5}$ & 0.10766 & 0.10965 & 0.10885 \\
$\beta_{3,6}$ & 0.11517 & 0.11715 & 0.11635 \\
$\beta_{3,7}$ & 0.12424 & 0.12620 & 0.12542 \\
$\beta_{3,8}$ & 0.13487 & 0.13682 & 0.13604 \\
$\beta_{4,1}$ & 0.09436 & 0.09644 & 0.09561 \\
$\beta_{4,2}$ & 0.09796 & 0.10002 & 0.09920 \\
$\beta_{4,3}$ & 0.10781 & 0.10986 & 0.10904 \\
$\beta_{4,4}$ & 0.12391 & 0.12594 & 0.12513 \\
$\beta_{4,5}$ & 0.14626 & 0.14828 & 0.14747 \\
$\beta_{4,6}$ & 0.17486 & 0.17686 & 0.17606 \\
$\beta_{4,7}$ & 0.20971 & 0.21170 & 0.21090 \\
$\beta_{4,8}$ & 0.25081 & 0.25278 & 0.25200 \\
$\beta_{4,9}$ & 0.29816 & 0.30012 & 0.29934 \\
$\beta_{4,10}$ & 0.35176 & 0.35371 & 0.35293 \\
$\beta_{4,11}$ & 0.41161 & 0.41354 & 0.41277 \\
$\beta_{4,12}$ & 0.47771 & 0.47963 & 0.47886 \\
$\beta_{4,13}$ & 0.55006 & 0.55196 & 0.55120 \\
$\beta_{4,14}$ & 0.62866 & 0.63055 & 0.62979 \\
$\beta_{4,15}$ & 0.71351 & 0.71538 & 0.71463 \\
$\beta_{4,16}$ & 0.80461 & 0.80647 & 0.80573 \\
\hline & & &
\end{tabular}

Tables 3 and 4 present the greyscale parameters obtained upon collage coding the mean observation

$$
u^{*}(x)=\frac{1}{N} \sum_{k=1}^{N} u_{k}(x) \text {. }
$$

We see that the corresponding values in the tables agree increasingly well as the number of realizations increase. In all cases, for any fixed choices of $i$ and $j$, the value of $\alpha_{i, j}$ is the same across all simulations and across the two tables. In fractal imaging, the $\alpha$ parameters represent a contrast adjustment and the $\beta$ parameters represent a brightness adjustment. In this example, the different realizations differ only by a vertical shift, so the corresponding fractal codes differ only in the brightness parameters. We also observe that values in the tables agree to at least two decimal places with the IFSM parameters for the unnoised signal, which makes sense since the additive noise has mean zero. 
Table 3. Greyscale map parameters $\alpha_{i, j}$ for the mean observation in the case of constant additive noise.

\begin{tabular}{cccc}
\hline coefficient & 50 & 100 & 200 \\
\hline$\alpha_{1,1}$ & 0.25000 & 0.25000 & 0.25000 \\
$\alpha_{1,2}$ & 0.25730 & 0.25730 & 0.25730 \\
$\alpha_{2,1}$ & 0.06250 & 0.06250 & 0.06250 \\
$\alpha_{2,2}$ & 0.06982 & 0.06982 & 0.06982 \\
$\alpha_{2,3}$ & 0.07715 & 0.07715 & 0.07715 \\
$\alpha_{2,4}$ & 0.08447 & 0.08447 & 0.08447 \\
$\alpha_{3,1}$ & 0.01562 & 0.01562 & 0.01562 \\
$\alpha_{3,2}$ & 0.02295 & 0.02295 & 0.02295 \\
$\alpha_{3,3}$ & 0.03028 & 0.03028 & 0.03028 \\
$\alpha_{3,4}$ & 0.03760 & 0.03760 & 0.03760 \\
$\alpha_{3,5}$ & 0.04493 & 0.04493 & 0.04493 \\
$\alpha_{3,6}$ & 0.05226 & 0.05226 & 0.05226 \\
$\alpha_{3,7}$ & 0.05958 & 0.05958 & 0.05958 \\
$\alpha_{3,8}$ & 0.06691 & 0.06691 & 0.06691 \\
$\alpha_{4,1}$ & 0.00391 & 0.00391 & 0.00391 \\
$\alpha_{4,2}$ & 0.01124 & 0.01124 & 0.01124 \\
$\alpha_{4,3}$ & 0.01857 & 0.01857 & 0.01857 \\
$\alpha_{4,4}$ & 0.02590 & 0.02590 & 0.02590 \\
$\alpha_{4,5}$ & 0.03323 & 0.03323 & 0.03323 \\
$\alpha_{4,6}$ & 0.04056 & 0.04056 & 0.04056 \\
$\alpha_{4,7}$ & 0.04789 & 0.04789 & 0.04789 \\
$\alpha_{4,8}$ & 0.05523 & 0.05523 & 0.05523 \\
$\alpha_{4,9}$ & 0.06256 & 0.06256 & 0.06256 \\
$\alpha_{4,10}$ & 0.06989 & 0.06989 & 0.06989 \\
$\alpha_{4,11}$ & 0.07722 & 0.07722 & 0.07722 \\
$\alpha_{4,12}$ & 0.08455 & 0.08455 & 0.08455 \\
$\alpha_{4,13}$ & 0.09188 & 0.09188 & 0.09188 \\
$\alpha_{4,14}$ & 0.09921 & 0.09921 & 0.09921 \\
$\alpha_{4,15}$ & 0.10650 & 0.10650 & 0.10650 \\
$\alpha_{4,16}$ & 0.11390 & 0.11390 & 0.11390 \\
\hline & & &
\end{tabular}

Example: We repeat the preceding example, this time adding place-dependent noise to produce the $N$ realizations:

$$
u_{k}(x)=u(x)+v_{k}(x), \quad k=1, \ldots, N,
$$

where $u(x)=0.8 x^{2}+0.1$, as before, $\left|v_{k}(x)\right|<0.1$ for all $x$, and, for each fixed $x, v_{k}(x)$ are samples from the
Table 4. Greyscale map parameters $\beta_{i, j}$ for the mean observation in the case of constant additive noise.

\begin{tabular}{cccc}
\hline coefficient & 50 & 100 & 200 \\
\hline$\beta_{1,1}$ & 0.07105 & 0.07261 & 0.07199 \\
$\beta_{1,2}$ & 0.07157 & 0.07312 & 0.07250 \\
$\beta_{2,1}$ & 0.08881 & 0.09076 & 0.08998 \\
$\beta_{2,2}$ & 0.08948 & 0.09142 & 0.09065 \\
$\beta_{2,3}$ & 0.09054 & 0.09247 & 0.09170 \\
$\beta_{2,4}$ & 0.09200 & 0.09391 & 0.09315 \\
$\beta_{3,1}$ & 0.09325 & 0.09530 & 0.09448 \\
$\beta_{3,2}$ & 0.09451 & 0.09654 & 0.09573 \\
$\beta_{3,3}$ & 0.09733 & 0.09935 & 0.09854 \\
$\beta_{3,4}$ & 0.10171 & 0.10372 & 0.10292 \\
$\beta_{3,5}$ & 0.10766 & 0.10965 & 0.10885 \\
$\beta_{3,6}$ & 0.11517 & 0.11715 & 0.11635 \\
$\beta_{3,7}$ & 0.12424 & 0.12620 & 0.12542 \\
$\beta_{3,8}$ & 0.13487 & 0.13682 & 0.13604 \\
$\beta_{4,1}$ & 0.09436 & 0.09644 & 0.09561 \\
$\beta_{4,2}$ & 0.09796 & 0.10002 & 0.09920 \\
$\beta_{4,3}$ & 0.10781 & 0.10986 & 0.10904 \\
$\beta_{4,4}$ & 0.12391 & 0.12594 & 0.12513 \\
$\beta_{4,5}$ & 0.14626 & 0.14828 & 0.14747 \\
$\beta_{4,6}$ & 0.17486 & 0.17686 & 0.17606 \\
$\beta_{4,7}$ & 0.20971 & 0.21170 & 0.21090 \\
$\beta_{4,8}$ & 0.25081 & 0.25278 & 0.25200 \\
$\beta_{4,9}$ & 0.29816 & 0.30012 & 0.29934 \\
$\beta_{4,10}$ & 0.35176 & 0.35371 & 0.35293 \\
$\beta_{4,11}$ & 0.41161 & 0.41354 & 0.41277 \\
$\beta_{4,12}$ & 0.47771 & 0.47963 & 0.47886 \\
$\beta_{4,13}$ & 0.55006 & 0.55196 & 0.55120 \\
$\beta_{4,14}$ & 0.62866 & 0.63055 & 0.62979 \\
$\beta_{4,15}$ & 0.71351 & 0.71538 & 0.71463 \\
$\beta_{4,16}$ & 0.80461 & 0.80647 & 0.80573 \\
\hline & & & \\
\end{tabular}

re-scaled distribution $\mathscr{N}(0,0.01)$. The right picture in Fig. 1 presents ten realizations. The results are presented in Tables 5-8, with the first table presenting the means of the greyscale map parameters obtained by collage coding the realizations separately and the second table presenting the greyscale map parameters obtained by collage coding the mean observation, $u^{*}(x)$. 
Table 5. Greyscale map mean parameters $\alpha_{i, j}$ in the case of place-dependent additive noise with a normal distribution.

\begin{tabular}{cccc}
\hline coefficient & 50 & 100 & 200 \\
\hline$\alpha_{1,1}$ & 0.24783 & 0.24817 & 0.24656 \\
$\alpha_{1,2}$ & 0.25505 & 0.25544 & 0.25412 \\
$\alpha_{2,1}$ & 0.06121 & 0.06223 & 0.06291 \\
$\alpha_{2,2}$ & 0.06938 & 0.07046 & 0.07104 \\
$\alpha_{2,3}$ & 0.07708 & 0.07851 & 0.07958 \\
$\alpha_{2,4}$ & 0.08467 & 0.08460 & 0.08452 \\
$\alpha_{3,1}$ & 0.01528 & 0.01578 & 0.01762 \\
$\alpha_{3,2}$ & 0.02500 & 0.02240 & 0.02327 \\
$\alpha_{3,3}$ & 0.03001 & 0.03025 & 0.0279 \\
$\alpha_{3,4}$ & 0.03565 & 0.03681 & 0.03749 \\
$\alpha_{3,5}$ & 0.04367 & 0.04528 & 0.04503 \\
$\alpha_{3,6}$ & 0.05363 & 0.05390 & 0.05448 \\
$\alpha_{3,7}$ & 0.06107 & 0.05783 & 0.05697 \\
$\alpha_{3,8}$ & 0.06887 & 0.06726 & 0.06449 \\
$\alpha_{4,1}$ & 0.00145 & 0.00175 & 0.00103 \\
$\alpha_{4,2}$ & 0.01221 & 0.00770 & 0.00599 \\
$\alpha_{4,3}$ & 0.01686 & 0.02246 & 0.02139 \\
$\alpha_{4,4}$ & 0.02663 & 0.02713 & 0.02261 \\
$\alpha_{4,5}$ & 0.03320 & 0.02775 & 0.02702 \\
$\alpha_{4,6}$ & 0.03973 & 0.03700 & 0.04394 \\
$\alpha_{4,7}$ & 0.05133 & 0.05263 & 0.05698 \\
$\alpha_{4,8}$ & 0.05110 & 0.05331 & 0.05169 \\
$\alpha_{4,9}$ & 0.06367 & 0.06415 & 0.05847 \\
$\alpha_{4,10}$ & 0.07064 & 0.07165 & 0.07154 \\
$\alpha_{4,11}$ & 0.08009 & 0.08117 & 0.08127 \\
$\alpha_{4,12}$ & 0.08196 & 0.08116 & 0.08289 \\
$\alpha_{4,13}$ & 0.08840 & 0.08420 & 0.08868 \\
$\alpha_{4,14}$ & 0.09788 & 0.09744 & 0.09734 \\
$\alpha_{4,15}$ & 0.10892 & 0.11101 & 0.11136 \\
$\alpha_{4,16}$ & 0.11107 & 0.11086 & 0.10584 \\
\hline & & &
\end{tabular}

Table 6. Greyscale map mean parameters $\beta_{i, j}$ in the case of place-dependent additive noise with a normal distribution.

\begin{tabular}{cccc}
\hline coefficient & 50 & 100 & 200 \\
\hline$\beta_{1,1}$ & 0.07567 & 0.07569 & 0.07636 \\
$\beta_{1,2}$ & 0.07616 & 0.07611 & 0.07662 \\
$\beta_{2,1}$ & 0.09393 & 0.09383 & 0.09400 \\
$\beta_{2,2}$ & 0.09424 & 0.09417 & 0.09423 \\
$\beta_{2,3}$ & 0.09511 & 0.09500 & 0.09509 \\
$\beta_{2,4}$ & 0.09649 & 0.09693 & 0.09764 \\
$\beta_{3,1}$ & 0.09838 & 0.09843 & 0.09808 \\
$\beta_{3,2}$ & 0.09875 & 0.09962 & 0.09961 \\
$\beta_{3,3}$ & 0.10234 & 0.10268 & 0.10412 \\
$\beta_{3,4}$ & 0.10740 & 0.10717 & 0.10756 \\
$\beta_{3,5}$ & 0.11273 & 0.11245 & 0.11306 \\
$\beta_{3,6}$ & 0.11915 & 0.11994 & 0.12040 \\
$\beta_{3,7}$ & 0.12841 & 0.12973 & 0.13083 \\
$\beta_{3,8}$ & 0.13923 & 0.14004 & 0.14117 \\
$\beta_{4,1}$ & 0.10043 & 0.09999 & 0.09986 \\
$\beta_{4,2}$ & 0.10254 & 0.10497 & 0.10664 \\
$\beta_{4,3}$ & 0.11348 & 0.11149 & 0.11200 \\
$\beta_{4,4}$ & 0.12806 & 0.12842 & 0.13100 \\
$\beta_{4,5}$ & 0.15160 & 0.15332 & 0.15423 \\
$\beta_{4,6}$ & 0.18088 & 0.18174 & 0.17955 \\
$\beta_{4,7}$ & 0.21230 & 0.21107 & 0.20814 \\
$\beta_{4,8}$ & 0.25745 & 0.25764 & 0.25772 \\
$\beta_{4,9}$ & 0.30314 & 0.30231 & 0.30508 \\
$\beta_{4,10}$ & 0.35598 & 0.35589 & 0.35696 \\
$\beta_{4,11}$ & 0.41463 & 0.41407 & 0.41363 \\
$\beta_{4,12}$ & 0.48285 & 0.48257 & 0.48226 \\
$\beta_{4,13}$ & 0.55575 & 0.55723 & 0.55612 \\
$\beta_{4,14}$ & 0.63338 & 0.63320 & 0.63350 \\
$\beta_{4,15}$ & 0.71795 & 0.71599 & 0.71530 \\
$\beta_{4,16}$ & 0.81047 & 0.81016 & 0.81133 \\
\hline & & &
\end{tabular}


Table 7. Greyscale map parameters $\alpha_{i, j}$ for the mean observation in the case of place-dependent additive noise with a normal distribution.

\begin{tabular}{cccc}
\hline coefficient & 50 & 100 & 200 \\
\hline$\alpha_{1,1}$ & 0.24995 & 0.25030 & 0.24878 \\
$\alpha_{1,2}$ & 0.25722 & 0.25759 & 0.25626 \\
$\alpha_{2,1}$ & 0.06152 & 0.06250 & 0.06312 \\
$\alpha_{2,2}$ & 0.06984 & 0.07086 & 0.07153 \\
$\alpha_{2,3}$ & 0.07725 & 0.07870 & 0.07967 \\
$\alpha_{2,4}$ & 0.08506 & 0.08504 & 0.08488 \\
$\alpha_{3,1}$ & 0.01534 & 0.01568 & 0.01744 \\
$\alpha_{3,2}$ & 0.02503 & 0.02235 & 0.02321 \\
$\alpha_{3,3}$ & 0.03006 & 0.03031 & 0.02814 \\
$\alpha_{3,4}$ & 0.03564 & 0.03679 & 0.03740 \\
$\alpha_{3,5}$ & 0.04381 & 0.04543 & 0.04513 \\
$\alpha_{3,6}$ & 0.05365 & 0.05386 & 0.05433 \\
$\alpha_{3,7}$ & 0.06127 & 0.05801 & 0.05702 \\
$\alpha_{3,8}$ & 0.06899 & 0.06750 & 0.06477 \\
$\alpha_{4,1}$ & 0.00143 & 0.00178 & 0.00084 \\
$\alpha_{4,2}$ & 0.01229 & 0.00781 & 0.00629 \\
$\alpha_{4,3}$ & 0.01675 & 0.02249 & 0.02141 \\
$\alpha_{4,4}$ & 0.02668 & 0.02705 & 0.02266 \\
$\alpha_{4,5}$ & 0.03321 & 0.02780 & 0.02719 \\
$\alpha_{4,6}$ & 0.03973 & 0.03692 & 0.04373 \\
$\alpha_{4,7}$ & 0.05143 & 0.05280 & 0.05710 \\
$\alpha_{4,8}$ & 0.05118 & 0.05356 & 0.05175 \\
$\alpha_{4,9}$ & 0.06371 & 0.06419 & 0.05859 \\
$\alpha_{4,10}$ & 0.07061 & 0.07159 & 0.07149 \\
$\alpha_{4,11}$ & 0.08008 & 0.08115 & 0.08136 \\
$\alpha_{4,12}$ & 0.08206 & 0.08141 & 0.08320 \\
$\alpha_{4,13}$ & 0.08855 & 0.08437 & 0.08873 \\
$\alpha_{4,14}$ & 0.09792 & 0.09758 & 0.09738 \\
$\alpha_{4,15}$ & 0.10897 & 0.11110 & 0.11133 \\
$\alpha_{4,16}$ & 0.11104 & 0.11083 & 0.10592 \\
\hline & & &
\end{tabular}

Example: We repeat the preceding example a last time, with the place-dependent noise $v_{k}(x)$ being samples from a beta distribution $\beta(1,2)$. The results are presented in Tables 9-12, with the first table presenting the means of the greyscale map parameters
Table 8. Greyscale map parameters $\beta_{i, j}$ for the mean observation in the case of place-dependent additive noise with a normal distribution.

\begin{tabular}{cccc}
\hline coefficient & 50 & 100 & 200 \\
\hline$\beta_{1,1}$ & 0.07489 & 0.07491 & 0.07554 \\
$\beta_{1,2}$ & 0.07536 & 0.07532 & 0.07583 \\
$\beta_{2,1}$ & 0.09382 & 0.09373 & 0.09392 \\
$\beta_{2,2}$ & 0.09407 & 0.09402 & 0.09405 \\
$\beta_{2,3}$ & 0.09504 & 0.09493 & 0.09505 \\
$\beta_{2,4}$ & 0.09634 & 0.09677 & 0.09751 \\
$\beta_{3,1}$ & 0.09837 & 0.09847 & 0.09814 \\
$\beta_{3,2}$ & 0.09874 & 0.09964 & 0.09963 \\
$\beta_{3,3}$ & 0.10232 & 0.10265 & 0.10403 \\
$\beta_{3,4}$ & 0.10740 & 0.10717 & 0.10759 \\
$\beta_{3,5}$ & 0.11268 & 0.11239 & 0.11303 \\
$\beta_{3,6}$ & 0.11914 & 0.11996 & 0.12046 \\
$\beta_{3,7}$ & 0.12833 & 0.12967 & 0.13081 \\
$\beta_{3,8}$ & 0.13918 & 0.13996 & 0.14106 \\
$\beta_{4,1}$ & 0.10045 & 0.10002 & 0.09993 \\
$\beta_{4,2}$ & 0.10251 & 0.10494 & 0.10653 \\
$\beta_{4,3}$ & 0.11352 & 0.1147 & 0.11200 \\
$\beta_{4,4}$ & 0.12803 & 0.12844 & 0.13098 \\
$\beta_{4,5}$ & 0.15160 & 0.15330 & 0.15416 \\
$\beta_{4,6}$ & 0.18087 & 0.18177 & 0.17964 \\
$\beta_{4,7}$ & 0.21227 & 0.21101 & 0.20810 \\
$\beta_{4,8}$ & 0.25742 & 0.25755 & 0.25768 \\
$\beta_{4,9}$ & 0.30313 & 0.30230 & 0.30504 \\
$\beta_{4,10}$ & 0.35599 & 0.35590 & 0.35697 \\
$\beta_{4,11}$ & 0.41464 & 0.41408 & 0.41360 \\
$\beta_{4,12}$ & 0.48282 & 0.48248 & 0.48216 \\
$\beta_{4,13}$ & 0.55569 & 0.55716 & 0.55611 \\
$\beta_{4,14}$ & 0.63338 & 0.63317 & 0.63351 \\
$\beta_{4,15}$ & 0.71793 & 0.71595 & 0.71530 \\
$\beta_{4,16}$ & 0.81049 & 0.81017 & 0.81130 \\
\hline & & & \\
& & &
\end{tabular}

obtained by collage coding the realizations separately and the second table presenting the greyscale map parameters obtained by collage coding the mean observation, $u^{*}(x)$. 
Table 9. Greyscale map mean parameters $\alpha_{i, j}$ in the case of place-dependent additive noise with a beta distribution.

\begin{tabular}{cccc}
\hline coefficient & 50 & 100 & 200 \\
\hline$\alpha_{1,1}$ & 0.25000 & 0.25000 & 0.25000 \\
$\alpha_{1,2}$ & 0.25732 & 0.25732 & 0.25732 \\
$\alpha_{2,1}$ & 0.06250 & 0.06250 & 0.06250 \\
$\alpha_{2,2}$ & 0.06983 & 0.06983 & 0.06983 \\
$\alpha_{2,3}$ & 0.07715 & 0.07715 & 0.07715 \\
$\alpha_{2,4}$ & 0.08447 & 0.08447 & 0.08447 \\
$\alpha_{3,1}$ & 0.01563 & 0.01563 & 0.01563 \\
$\alpha_{3,2}$ & 0.02295 & 0.02295 & 0.02295 \\
$\alpha_{3,3}$ & 0.03028 & 0.03028 & 0.03028 \\
$\alpha_{3,4}$ & 0.03760 & 0.03760 & 0.03760 \\
$\alpha_{3,5}$ & 0.04493 & 0.04493 & 0.04493 \\
$\alpha_{3,6}$ & 0.05226 & 0.05226 & 0.05226 \\
$\alpha_{3,7}$ & 0.05958 & 0.05958 & 0.05958 \\
$\alpha_{3,8}$ & 0.06691 & 0.06691 & 0.06691 \\
$\alpha_{4,1}$ & 0.00391 & 0.00391 & 0.00391 \\
$\alpha_{4,2}$ & 0.01124 & 0.01124 & 0.01124 \\
$\alpha_{4,3}$ & 0.01857 & 0.01857 & 0.01857 \\
$\alpha_{4,4}$ & 0.02590 & 0.02590 & 0.02590 \\
$\alpha_{4,5}$ & 0.03323 & 0.03323 & 0.03323 \\
$\alpha_{4,6}$ & 0.04056 & 0.04056 & 0.04056 \\
$\alpha_{4,7}$ & 0.04790 & 0.04790 & 0.04790 \\
$\alpha_{4,8}$ & 0.05523 & 0.05523 & 0.05523 \\
$\alpha_{4,9}$ & 0.06256 & 0.06256 & 0.06256 \\
$\alpha_{4,10}$ & 0.06989 & 0.06989 & 0.06989 \\
$\alpha_{4,11}$ & 0.07722 & 0.07722 & 0.07722 \\
$\alpha_{4,12}$ & 0.08455 & 0.08455 & 0.08455 \\
$\alpha_{4,13}$ & 0.09188 & 0.09188 & 0.09188 \\
$\alpha_{4,14}$ & 0.09921 & 0.09921 & 0.09921 \\
$\alpha_{4,15}$ & 0.10655 & 0.10655 & 0.10655 \\
$\alpha_{4,16}$ & 0.11388 & 0.11388 & 0.11388 \\
\hline & & &
\end{tabular}

Table 10. Greyscale map mean parameters $\beta_{i, j}$ in the case of place-dependent additive noise with a beta distribution.

\begin{tabular}{cccc}
\hline coefficient & 50 & 100 & 200 \\
\hline$\beta_{1,1}$ & 0.27162 & 0.28736 & 0.31950 \\
$\beta_{1,2}$ & 0.27019 & 0.28577 & 0.31760 \\
$\beta_{2,1}$ & 0.33953 & 0.35920 & 0.39937 \\
$\beta_{2,2}$ & 0.33824 & 0.35776 & 0.39762 \\
$\beta_{2,3}$ & 0.33735 & 0.35671 & 0.39625 \\
$\beta_{2,4}$ & 0.33684 & 0.35605 & 0.39528 \\
$\beta_{3,1}$ & 0.35650 & 0.37716 & 0.41934 \\
$\beta_{3,2}$ & 0.35580 & 0.37630 & 0.41817 \\
$\beta_{3,3}$ & 0.35666 & 0.37701 & 0.41856 \\
$\beta_{3,4}$ & 0.35909 & 0.37928 & 0.42052 \\
$\beta_{3,5}$ & 0.36308 & 0.38311 & 0.42404 \\
$\beta_{3,6}$ & 0.36863 & 0.38851 & 0.42912 \\
$\beta_{3,7}$ & 0.37574 & 0.39547 & 0.43577 \\
$\beta_{3,8}$ & 0.38441 & 0.40399 & 0.44397 \\
$\beta_{4,1}$ & 0.36075 & 0.38165 & 0.42433 \\
$\beta_{4,2}$ & 0.36239 & 0.38313 & 0.42550 \\
$\beta_{4,3}$ & 0.37028 & 0.39087 & 0.43292 \\
$\beta_{4,4}$ & 0.38442 & 0.40485 & 0.44660 \\
$\beta_{4,5}$ & 0.40481 & 0.42509 & 0.46652 \\
$\beta_{4,6}$ & 0.43145 & 0.45158 & 0.49269 \\
$\beta_{4,7}$ & 0.46434 & 0.48431 & 0.52511 \\
$\beta_{4,8}$ & 0.50348 & 0.52330 & 0.56378 \\
$\beta_{4,9}$ & 0.54887 & 0.56853 & 0.60870 \\
$\beta_{4,10}$ & 0.60051 & 0.62002 & 0.65988 \\
$\beta_{4,11}$ & 0.65840 & 0.67776 & 0.71730 \\
$\beta_{4,12}$ & 0.72254 & 0.74174 & 0.78097 \\
$\beta_{4,13}$ & 0.79293 & 0.81198 & 0.85089 \\
$\beta_{4,14}$ & 0.86957 & 0.88846 & 0.92706 \\
$\beta_{4,15}$ & 0.95245 & 0.97120 & 1.00950 \\
$\beta_{4,16}$ & 1.04160 & 1.06020 & 1.09820 \\
\hline & & &
\end{tabular}


Table 11. Greyscale map parameters $\alpha_{i, j}$ for the mean observation in the case of place-dependent additive noise with a beta distribution.

\begin{tabular}{cccc}
\hline coefficient & 50 & 100 & 200 \\
\hline$\alpha_{1,1}$ & 0.25000 & 0.28736 & 0.25000 \\
$\alpha_{1,2}$ & 0.25732 & 0.28577 & 0.25732 \\
$\alpha_{2,1}$ & 0.06250 & 0.35920 & 0.06250 \\
$\alpha_{2,2}$ & 0.06983 & 0.35776 & 0.06983 \\
$\alpha_{2,3}$ & 0.07715 & 0.35671 & 0.07715 \\
$\alpha_{2,4}$ & 0.08447 & 0.35605 & 0.08447 \\
$\alpha_{3,1}$ & 0.01563 & 0.37716 & 0.01563 \\
$\alpha_{3,2}$ & 0.02295 & 0.37630 & 0.02295 \\
$\alpha_{3,3}$ & 0.03028 & 0.37701 & 0.03028 \\
$\alpha_{3,4}$ & 0.03760 & 0.37928 & 0.03760 \\
$\alpha_{3,5}$ & 0.04493 & 0.38311 & 0.04493 \\
$\alpha_{3,6}$ & 0.05226 & 0.38851 & 0.05226 \\
$\alpha_{3,7}$ & 0.05958 & 0.39547 & 0.05958 \\
$\alpha_{3,8}$ & 0.06691 & 0.40399 & 0.06691 \\
$\alpha_{4,1}$ & 0.00391 & 0.38165 & 0.00391 \\
$\alpha_{4,2}$ & 0.01124 & 0.38313 & 0.01124 \\
$\alpha_{4,3}$ & 0.01857 & 0.39087 & 0.01857 \\
$\alpha_{4,4}$ & 0.02590 & 0.40485 & 0.02590 \\
$\alpha_{4,5}$ & 0.03323 & 0.42509 & 0.03323 \\
$\alpha_{4,6}$ & 0.04056 & 0.45158 & 0.04056 \\
$\alpha_{4,7}$ & 0.04790 & 0.48431 & 0.04790 \\
$\alpha_{4,8}$ & 0.05523 & 0.52330 & 0.05523 \\
$\alpha_{4,9}$ & 0.06256 & 0.56853 & 0.06256 \\
$\alpha_{4,10}$ & 0.06989 & 0.62002 & 0.06989 \\
$\alpha_{4,11}$ & 0.07722 & 0.67776 & 0.07722 \\
$\alpha_{4,12}$ & 0.08455 & 0.74174 & 0.08455 \\
$\alpha_{4,13}$ & 0.09188 & 0.81198 & 0.09188 \\
$\alpha_{4,14}$ & 0.09921 & 0.88846 & 0.09921 \\
$\alpha_{4,15}$ & 0.10655 & 0.97120 & 0.10655 \\
$\alpha_{4,16}$ & 0.11388 & 1.06020 & 0.11388 \\
\hline & & &
\end{tabular}

We remark that, in all examples, the mean function $u^{*}(x)$ constructed from the $N$ observations may be viewed as a kind of denoising of the noisy signals.

In closing, we mention that the extension of the ideas in this paper to images (as opposed to signals) is the focus of some current work. We mention here that the ideas link naturally to the idea of image denoising, which has been the focus of much work in image science, including work from a fractal-based direction Ghazel et al. (2003).

\section{REFERENCES}

Barnsley MF, Ervin V, Hardin D, Lancaster J (1985). Solution of an inverse problem for fractals and other
Table 12. Greyscale map parameters $\beta_{i, j}$ for the mean observation in the case of place-dependent additive noise with a beta distribution.

\begin{tabular}{cccc}
\hline coefficient & 50 & 100 & 200 \\
\hline$\beta_{1,1}$ & 0.27162 & 0.28736 & 0.31950 \\
$\beta_{1,2}$ & 0.27019 & 0.28577 & 0.31760 \\
$\beta_{2,1}$ & 0.33953 & 0.35920 & 0.39937 \\
$\beta_{2,2}$ & 0.33824 & 0.35776 & 0.39762 \\
$\beta_{2,3}$ & 0.33735 & 0.35671 & 0.39625 \\
$\beta_{2,4}$ & 0.33684 & 0.35605 & 0.39528 \\
$\beta_{3,1}$ & 0.35650 & 0.37716 & 0.41934 \\
$\beta_{3,2}$ & 0.35580 & 0.37630 & 0.41817 \\
$\beta_{3,3}$ & 0.35666 & 0.37701 & 0.41856 \\
$\beta_{3,4}$ & 0.35909 & 0.37928 & 0.42052 \\
$\beta_{3,5}$ & 0.36308 & 0.38311 & 0.42404 \\
$\beta_{3,6}$ & 0.36863 & 0.38851 & 0.42912 \\
$\beta_{3,7}$ & 0.37574 & 0.39547 & 0.43577 \\
$\beta_{3,8}$ & 0.38441 & 0.40399 & 0.44397 \\
$\beta_{4,1}$ & 0.36075 & 0.38165 & 0.42433 \\
$\beta_{4,2}$ & 0.36239 & 0.38313 & 0.42550 \\
$\beta_{4,3}$ & 0.37028 & 0.39087 & 0.43292 \\
$\beta_{4,4}$ & 0.38442 & 0.40485 & 0.44660 \\
$\beta_{4,5}$ & 0.40481 & 0.42509 & 0.46652 \\
$\beta_{4,6}$ & 0.43145 & 0.45158 & 0.49269 \\
$\beta_{4,7}$ & 0.46434 & 0.48431 & 0.52511 \\
$\beta_{4,8}$ & 0.50348 & 0.52330 & 0.56378 \\
$\beta_{4,9}$ & 0.54887 & 0.56853 & 0.60870 \\
$\beta_{4,10}$ & 0.60051 & 0.62002 & 0.65988 \\
$\beta_{4,11}$ & 0.65840 & 0.67776 & 0.71730 \\
$\beta_{4,12}$ & 0.72254 & 0.74174 & 0.78097 \\
$\beta_{4,13}$ & 0.79293 & 0.81198 & 0.85089 \\
$\beta_{4,14}$ & 0.86957 & 0.88846 & 0.92706 \\
$\beta_{4,15}$ & 0.95245 & 0.97120 & 1.00950 \\
$\beta_{4,16}$ & 1.04160 & 1.06020 & 1.09820 \\
\hline & & &
\end{tabular}

sets. Proc Nat Acad Sci USA 83:1975-77.

Barnsley MF (1989). Fractals everywhere. New York: Academic Press.

Barnsley MF, Demko S (1985). Iterated function systems and the global construction of fractals. Proc Roy Soc London Ser A 399:243-75.

Barnsley MF, Hurd L (1993). Fractal image compression. Massachussetts: A.K. Peters.

Bharucha-Reid AT (1972). Random integral equations. Mathematics in Science and Engineering. 96, New York: Academic Press.

Centore P, Vrscay ER (1994). Continuity of attractors and invariant measures for Iterated Function Systems. Canad Math Bull 37(3):315-29. 
Fisher Y(1995). Fractal image compression, theory and application. New York: Springer-Verlag.

Forte B, Vrscay ER (1995). Solving the inverse problem for function and image approximation using iterated function systems. In: Dynamics of Continuous, Discrete and Impulsive Systems 1(2).

Forte B, Vrscay ER (1999). Theory of generalized fractal transforms. In: Fisher Y, ed. Fractal Image Encoding and Analysis. NATO ASI Series F. Vol. 159. New York: Springer Verlag.

Ghazel M, Freeman GH and Vrscay ER (2003). Fractal image denoising. IEEE Trans Image Proc 12(12):1560-78.

Hanš O (1957). Reduzierende zufallige Transformationen. Czechoslovak Math J 7(82):154-8.

Himmelberg CJ (1975). Measurable relations. Fund Math $87: 53-72$.

Hutchinson J (1981). Fractals and self-similarity. Indiana Univ J Math 30:713-47.

Iacus S, La Torre D (2005). A comparative simulation study on the IFS distribution function estimator. Nonlinear Anal Real World Appl 6(5):858-73.

Iacus S, La Torre D (2005). Approximating distribution functions by iterated function systems. J Appl Math Dec Sci 1:33-46.

Itoh S (1977). A random fixed point theorem for a multivalued contraction mapping. Pacific J Math 68(1):85-90.

Itoh S (1979). Random fixed point theorems with an application to random differential equations in Banach spaces. J Math Anal App 67:261-73.

Kunze H, La Torre D, Vrscay ER (2008). From iterated function systems to iterated multifunction systems.
Commun Appl Nonlinear Anal 15(4):1-15.

La Torre D, Vrscay ER, Ebrahimi M, Barnsley M (2009). Measure-valued images, associated fractal transforms and the affine self-similarity of images. SIAM J Imaging Sci 2(2):470-507.

La Torre D, Vrscay ER (2011). Generalized fractal transforms and self-similarity: recent results and applications. Image Anal Stereol 30(2):63-76.

Lin TC (1995). Random approximations and random fixed point theorems for continuous 1-set-contractive random maps. Proc Amer Math Soc 123(4):1167-76.

Liu LS (1997). Some random approximations and random fixed point theorems for 1-set-contractive random operators. Proc Amer Math Soc 125(2):515-21.

Lu N (2003). Fractal imaging. New York: Academic Press.

Papageorgiou NS (1988). Random fixed points and random differential inclusions. Int J Math Sci 11(3):551-59.

Shahzad N (2001). Random fixed points of set-valued maps. Nonlinear Anal 45(6):689-92.

Shahzad N (2004). Random fixed points of pseudocontractive random operators. J Math Anal Appl 296(1):302-8.

Shahzad N (2004). Random fixed points of $K$-set and pseudo-contractive random maps. Nonlinear Anal 57(2):173-81.

Spaček A (1955). Zufallige Gleichungen. Czechoslovak Math J 5(80):462-66.

Vrscay ER, Saupe D (1999). Can one break the 'collage barrier' in fractal image coding? In: Dekking M, LevyVehel J, Lutton E, Tricot C eds. Fractals: Theory and Applications in Engineering, London: Springer-Verlag. 307-23. 